\title{
NEUROMUSCULAR DISORDERS
}

\section{LIMB-GIRDLE MUSCULAR DYSTROPHIES}

The phenotype in limb-girdle muscular dystrophy (LGMD) type 21 was defined by mutation analysis, protein studies, and respiratory and cardiac involvement studied in 16 patients from 14 families with fukutin-related protein (FKRP) gene mutations and LGMD, at the Institute of Human Genetics, University Newcastle upon Tyne, UK. The age at onset ranged from 2 to 40 years (mean 19.2 years). Five patients had symptoms in childhood, including a peculiar gait, inability to run, muscle cramps, and myalgia. The remaining 11 patients had onset of symptoms in the $2^{\text {nd }}$ to $4^{\text {th }}$ decades. The predominant mode of presentation was a waddling gait, and difficulties in climbing stairs. Thirteen adults were homozygous for the common C826A mutation in FKRP. Three cases, 2 presenting in childhood with more progressive disease, were compound heterozygotes for C826A. Muscle involvement, including calf hypertrophy, was similar to dystrophinopathy, with proximal weakness. Patients with LGMD21 had cardiac complications (6 cases), and respiratory involvement, including the diaphragm (10 cases). Serum creatine kinase in all patients was 5 to 70 times normal. Muscle biopsies showed a reduction of the protein laminin a2 immunolabeling. LGMD21 due to FKRP mutations is a common cause of LGMD, and patients frequently suffered respiratory and cardiac complications. (Poppe M, Cree L, Bourke J, et al. The phenotype of limb-girdle muscular dystrophy type 21 . Neurology April (2 of 2) 2003;60:1246-1251). (Reprints: Professor Kate Bushby, Professor of Neuromuscular Genetics, Institute of Human Genetics, International Centre for Life, Central Parkway, Newcastle upon Tyne NE1 3BZ, UK).

COMMENT. Fifteen genetic subtypes of limb-girdle muscular dystrophy (LGMD) are recognized and identified by mutation analysis. Five are autosomal dominant and 10 autosomal recessive. LGMD21 is due to fukutin-related protein gene mutations and is a relatively common cause of LGMD. As indicated by Wicklund MP and Hilton-Jones D, in their editorial (Neurology April (2 of 2) 2003;60:1230-1231), early identification may diminish associated morbidity from respiratory and cardiac complications.

Caveolin-3 gene mutations may cause the progressive LGMD type 1C and rippling muscle disease.

\section{CAVEOLIN-3 MUTATIONS IN RIPPLING MUSCLE DISEASE}

Two unrelated patients with novel homozygous missense mutations (L86P and A92T) in caveolin-3 gene (CAV3), presenting with a severe form of rippling muscle disease (RMD), are reported from the University of Bonn, and other centers in Germany. Patient 1 had severe muscle stiffness from early childhood and contractures of the Achilles tendons. Patient 2 had slowly progressive muscle weakness beginning in early adulthood. Symptoms were restricted to skeletal muscles, and heart muscle was not affected. (Kubisch C, Schoser BGH, v During M, et al. Homozygous mutations in Caveolin-3 cause a severe form of rippling muscle disease. Ann Neurol April 2003;53:512-520). (Respond: Dr 
Matthias Vorgerd, Department of Neurology, Ruhr-University Bochum, Buerkle-de-laCamp-Platz 1, D-44789 Bochum, Germany).

COMMENT. Hereditary rippling muscle disease (RMD) is a rare autosomal dominant, nonprogressive myopathy characterized by increased muscle irritability (percussion-induced contractions), electrically silent wave-like contractions (rippling muscle), and muscle mounding with percussion. The above authors have previously reported mutations in CAV-3 in families with RMD and in one sporadic case of RMD. CAV-3 mutations may also occur in limb-girdle muscular dystrophy 1C, distal myopathy, and in children with elevated CK without myopathy.

\section{LONG-TERM OUTCOME IN GUILLAIN-BARRE SYNDROME}

The long-term sequelae and early predictors of sequelae were determined in a chart review of 47 children with Guillain-Barre syndrome (GBS) of whom 30 had been treated with intravenous gammaglobulin (IVIG) at The Hospital for Sick Children, Toronto, Canada. Persisting long-term muscle weakness at least 2 years after recovery was present in $23 \%$ of cases. Weakness was mild and patients were functionally independent. Risk factors for long-term muscle weakness were a young age at onset and a rapid progression to maximal weakness in the acute illness $(\mathrm{P}=0.03$ ). (Vajsar J, Fehlings $\mathrm{D}$, Stephens D. Long-term outcome in children with Guillain-Barre syndrome. J Pediatr March 2003;142:305-309). (Reprints: Jiri Vajsar MD MSc, Division of Neurology, The Hospital for Sick Children, 555 University Ave, Toronto, Ontario M5G 1X8, Canada).

COMMENT. Age associated changes in the symptomatology of GBS in children have been reported previously (Sakakihara Y, Kamoshita S. 1991, see Progress in Poediatric Neurology II, PNB Publ, 1994;pp271-2). Children between 3 and 9 years are most susceptible, and the total duration of the illness is longer in younger children. Bulbar nerves are most frequently affected in the younger age group, and the facial nerve in older patients. The stage of myelinogenic maturation might explain the age-associated changes in symptomatology of GBS.

\section{ATTENTION DEFICIT DISORDER}

\section{OROS METHYLPHENIDATE LONG-TERM STUDY IN ADHD}

Once-daily osmotic controlled-release (OROS) methylphenidate (Concerta) in the treatment of 407 children, aged 6-13 years, with ADHD who were known MPH responders, was evaluated in a 24-month multicenter, open-label, nonrandomized study, and reported from the Massachusetts General Hospital, Boston. In $289(71 \%)$ who completed 12 months'treatment, OROS MPH was well tolerated and effectiveness was maintained up to 12 months. Of 118 who failed to complete the trial period, $31(7.6 \%)$ discontinued for lack of effectiveness, and $28(6.9 \%)$ for adverse events, including tics (7), and insomnia (5). New onset of tics occurred in 23 (6.4\%) subjects. (Wilens T, Pelham W, Stein M, et al. J Am Acad Child Adolesc Psychiatry April 2003;42:424-433). 